\title{
Endovascular repair of type B aortic dissection: a study by computational fluid dynamics
}

\author{
Yi Fan', Stephen Wing-Keung Cheng ${ }^{2}$, Kai-Xiong Qing ${ }^{2}$, Kwok-Wing Chow ${ }^{1}$ *
}

\author{
${ }^{1}$ Department of Mechanical Engineering, University of Hong Kong, Pokfulam, Hong Kong; \\ ${ }^{2}$ Department of Surgery, University of Hong Kong, Pokfulam, Hong Kong. \\ Email: "kwchow@,hkusua.hku.hk
}

Received 7 July 2010; revised 19 July 2010; accepted 22 July 2010.

\begin{abstract}
Aortic dissection is a dangerous pathological condition where blood intrudes into the layers of the arterial walls, creating an artificial channel (false lumen). In the absence of thrombosis or surgical intervention, blood will enter the false lumen through the proximal tear, and join the true lumen again through a distal tear. Rupture of the weakened outer wall will result in extremely high mortality rates. Type $B$ thoracic aortic dissection (TAD), occurring along the descending aorta, can be repaired surgically by the deployment of an endovascular stent graft, concealing the proximal entry tear. Blood might still flow into the false lumen (FL) through the distal tear. The domain of such flow should be minimized, as complete thrombosis of the FL is generally believed to be more beneficial for the patient. The dependence on the area ratios of the lumens and size of these tears is studied by computational fluid dynamics.
\end{abstract}

Keywords: Aortic Dissection; Endovascular Repair; Stent Graft; Computational Fluid Dynamics

\section{INTRODUCTION}

Techniques and principles of continuum mechanics, especially those of computational fluid dynamics (CFD), have been used with increasing popularity in analyzing the characteristics and diseases of the cardiovascular system [1-5], e.g. stenosis, aneurysms and dissection [6-9]. The main objective here is to employ CFD to examine one particular pathological configuration, namely, thoracic aortic dissection (TAD). TAD is a dangerous condition, whereby tears along the ascending or descending aorta generate artificial channels (false lumens) of blood flow [10-14]. Untreated TAD may lead to rupture of the vessels, and results in high mortality rates for the patients.

In the terminology of clinical medicine (Stanford classification scheme), TAD is termed type A / B if the dissection occurs along the ascending / descending aorta respectively, with the latter usually associated with a higher survival rate. For type B TAD, further subdivision to acute and chronic regimes is based on the time scales of development. The primary goal of the present work is to examine the various biomechanical factors governing the management strategy and outcome of patients with type B TAD.

A patient with untreated TAD will be vulnerable to several dangerous consequences. A large volume of blood flows through the proximal tear into a false lumen with weakened walls [13], and may rejoin the original vessel, the true lumen, through a distal tear in the abdominal region. The size and location of the tears will affect the flow dynamics. Partial thrombosis may occlude the distal tear, creating an even more desirable situation. Indeed the relationship between the degree of patency of the false lumen and the survival rate of patients has been studied intensively [14].

The traditional treatment is open surgery. Recently, an important alternative is endovascular repair, whereby a stent graft is deployed to conceal the entry tear $[11,12]$. The advantage is shorter recovery time, and thus the period of hospital stay is reduced. However, many clinical and scientific issues arise accordingly. A very critical one is the degree of thrombosis, or termed from the opposite perspective, the level of patency of the false lumen (FL). Studies show that FL with complete thrombosis is usually associated with lower risk, while patients with a fully patent FL can expect increased chance of aortic rupture and death. We shall not attempt to address the issue of partially patent false lumen in this work.

Methods of computational fluid dynamics will be employed to investigate the effects of these dynamic, as well as geometric, factors on the post-operation risk analysis of endovascular stent graft deployment. More precisely, the critical features to be studied are:

a) the ratio of the area of the false lumen to that of the true lumen; 
b) the size of the re-entry tear;

c) the position of the re-entry tear with respect to the aortic arch.

The extent of thrombosis will be assessed by determining the domains of negligible fluid flows. The working assumption is that if the fluid does not display any motion, blood will undergo complete thrombosis in that domain. It is generally believed that a larger extent of thrombosis in the false lumen is beneficial to the patient.

The structure of this paper can now be explained. The technical details of the computations, i.e. the governing equations and the boundary conditions, are presented in Section 2. Numerical results from varying the various biomechanical factors are discussed in Section 3, followed by Conclusions in Section 4.

\section{METHODOLOGY}

Blood flow phenomena are generally very complex, combining the difficulties of nonlinear governing equations, irregular geometry and pulsatile pressure gradient. Recently, computational fluid dynamics (CFD) techniques have been employed frequently in biomedical engineering research. The dependence on the relevant biomechanical factors can be estimated through numerical simulations, as in vivo experimentations are usually difficult, time consuming, expensive or simply impossible.

Two pieces of CFD software are adopted in this study, namely, a pre-processor, GAMBIT 2, Fluent Inc., and a post-processor, FLUENT 6 (Fluent Inc.). GAMBIT provides a comprehensive set of tools in creating the geometry and high quality mesh within a relatively short period of time. FLUENT has been widely used in the computational biomedical engineering community. In addition to accuracy and efficiency, the post-processing feature generates high resolution images and animations.

\subsection{Modeling}

By applying a pre-processor tool (GAMBIT 2, Fluent Inc.), an aortic model can be constructed according to the dimensions of a contrast-enhanced, Computerized Tomography (CT) image of a patient with thoracic aortic dissection. Geometrically, the relevant length scales are the diameter of the vessel and the diameter of curvature of the aortic arch. Typical values of $30 \mathrm{~mm}$ and $93 \mathrm{~mm}$ will be employed for subsequent numerical simulations. The length of the ascending aorta which extends immediately from the aortic valve is taken to be $30 \mathrm{~mm}$. An idealized geometry, an aortic arch with constant diameter, consisting of the ascending aorta and the descending aorta, is thus adopted. The actual shape of the descending aorta with dissection will obviously be considerably more complicated than the idealized geometry selected here [15], but it is essential to capture the flow physics in a simplified model first.
Two chambers, the true and false lumens, are created by defining a portion of a circular cylinder (the intimal flap) with axis perpendicular to the outlet in the descending aorta (Figure 1(a)). This circular wall is created by

a) forming two circular arcs, and

b) linking them together to form a surface.

This surface is then swept to form a three dimensional curved surface. The thickness of the intimal flap is taken as $2 \mathrm{~mm}$.

Computations for the discrete cells (Figure 1(b)) are then performed. About 200,000 elements are created in the flow, enabling the governing equations to be solved in each of the smaller domains.

A vertical coordinate system must be introduced to measure position. We define $y=0$ as the location where the aortic arch begins (Figure 1(c)), and thus the reentry tear is typically located at negative values of $y$.

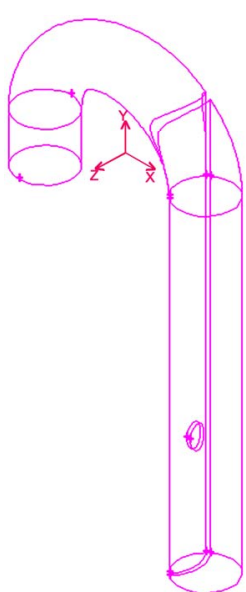

(a)

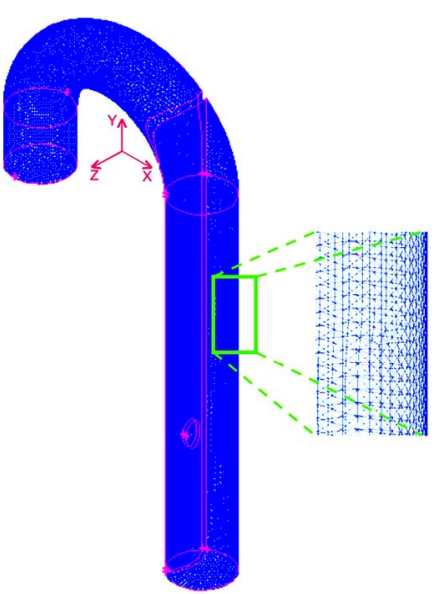

(b)

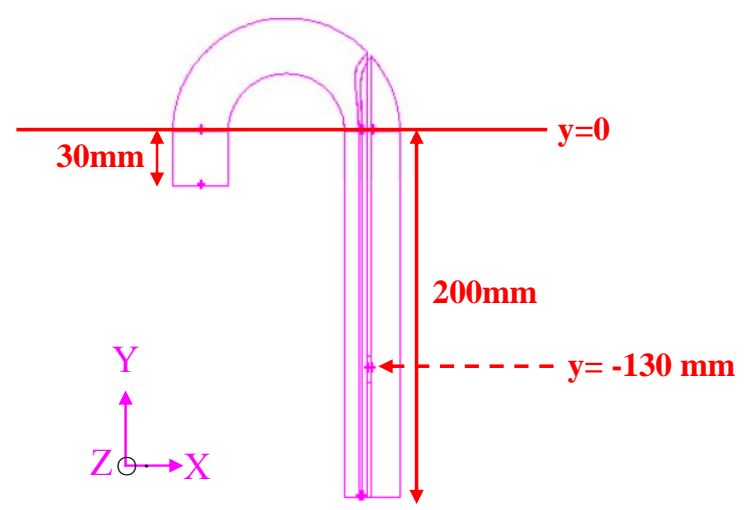

(c)

Figure 1. (a) Geometry of the aortic model; (b) Meshed aortic model; (c) Definition of the vertical coordinate scale; length of ascending aorta $=30 \mathrm{~mm}$, length of descending aorta $=200 \mathrm{~mm}$, the re-entry tear is located at the level $\mathrm{y}=$ $-130 \mathrm{~mm}$ while the entry tear is covered after endovascular repair. 
The descending aorta is thus divided into the true and false lumens. Endovascular repair is assumed to have been performed, and the proximal tear has been covered by the deployment of a stent graft. A re-entry tear, modeled by an elliptic hole of the intimal flap near the distal end, links the false and true lumens. Different area ratios of the lumens are obtained by varying the position of the circular boundary plane (Figures 2(a), 2(b)).

In practice, the false lumen is typically several times larger than the true lumen. In subsequent simulations, six models with different area ratios are employed.

\subsection{Governing Equations}

The governing equations of fluid motion are the usual continuity equation (conservation of mass), and the Navier-Stokes (NS) equations (rate of change of momentum). In tensor notations (repeated indices implying summation), the continuity equation is

$$
\frac{\partial u_{i}}{\partial x_{i}}=0,
$$

and the three dimensional NS equations are

$$
\frac{\partial u_{i}}{\partial t}+u_{j} \frac{\partial u_{i}}{\partial x_{j}}=-\frac{1}{\rho} \frac{\partial p}{\partial x_{i}}+\frac{1}{\rho} \frac{\partial \tau_{i j}}{\partial x_{i}},
$$

where $\rho=$ fluid density; $u_{i}(i=1,2,3)=$ components of velocity vector; $\tau_{i j}=$ normal and shear stresses; $p=$ pressure.
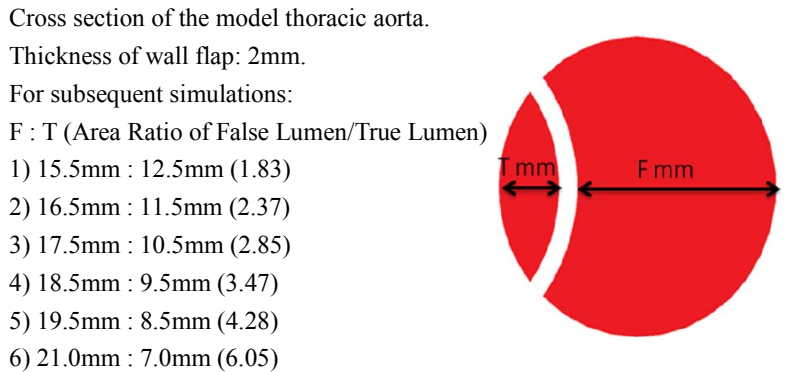

(a)

Re-entry (distal) tear of an elliptical shape. For subsequent simulations: Area (Long (L); Short (S)) axes

1) $113.2 \mathrm{~mm}^{2}(20 \mathrm{~mm} ; 14 \mathrm{~mm})$

2) $172.8 \mathrm{~mm}^{2}(22 \mathrm{~mm} ; 10 \mathrm{~mm})$

3) $216.8 \mathrm{~mm}^{2}(23 \mathrm{~mm} ; 12 \mathrm{~mm})$

4) $263.9 \mathrm{~mm}^{2}(24 \mathrm{~mm} ; 14 \mathrm{~mm})$

5) $306.3 \mathrm{~mm}^{2}(26 \mathrm{~mm} ; 15 \mathrm{~mm})$

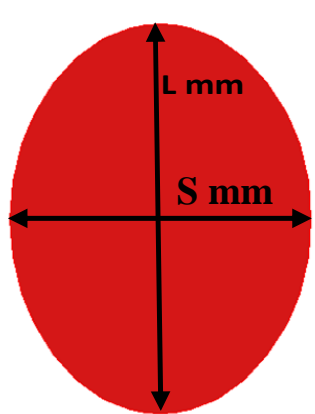

(b)

Figure 2. (a) Dimensions of the true and false lumens; (b) Size of the re-entry tear.
The finite volume technique is utilized. In the control volume generated by the pre-processor, these governing equations are discretized and solved iteratively considering the fully three dimensional character of the flow configuration.

\subsection{Boundary Conditions}

Several assumptions regarding the rheological properties of blood will be made. Although blood is a suspension of blood cells and platelets in plasma, only plasma will be taken into consideration here as particles are normally dynamical unimportant. The blood is thus treated as an incompressible, homogeneous Newtonian fluid. This is a reasonable assumption for large arteries like the thoracic aorta, supported by recent studies which confirmed that non-Newtonian effects in large arteries are small [1]. The density of blood, $\rho$, is accordingly taken as $1060 \mathrm{~kg}$ $\mathrm{m}^{-3}$ while the viscosity, $\mu$, is set as $0.0035 \mathrm{~N} \mathrm{~s} \mathrm{~m}^{-2}[2,3]$. The no slip boundary conditions are adopted. To reduce the complexity of the problem, the elasticity of the wall, including the intimal flap, [16] will be neglected and will be addressed in a future paper.

Similar to many earlier works in the literature, a velocity inlet and a pressure outlet are adopted as boundary conditions. To achieve realistic results, the velocity profiles and pressure waveforms are calibrated to match closely the experimentally measured values as a function of time. Pulsatile (pulsating) profiles are adopted for both the velocity and pressure (Figure 3). At a fixed time, the velocity and pressure are assumed to be uniform across the inlet and exit respectively. Applying the three-dimensional CFD codes in FLUENT 6 (Fluent Inc.), the blood flow pattern in the thoracic aorta can now be simulated.

A remark on some typical numbers is in order. The peak inflow rate is taken as about $0.0002 \mathrm{~m} \mathrm{~s}^{-1}$. The range of pressure at the outlet is $82 \mathrm{mmHg}-125 \mathrm{mmHg}$. The systolic cycle is taken as $1 \mathrm{~s}$. The mean velocity is $0.0309 \mathrm{~m} \mathrm{~s}^{-1}$ and the corresponding mean Reynolds number, $\operatorname{Re}=281$, and thus a laminar flow assumption is valid. Even with fairly arbitrary initial conditions, typically three cycles of computations will generate a periodic output, and data for the fourth cycle will be reported in the subsequent discussion.

Both the velocity and pressure are analyzed at the peak flow rate. We define a cut-off plane as the transverse planar surface in the descending aorta above which fluid motion does not exist, or at least is negligible. In clinical practice, the working assumption is that complete thrombosis will occur above this cut-off plane.

\section{COMPUTATIONAL RESULTS}

We shall now study several geometric factors which will 
affect the dynamics of the dissection and the management strategy for the patients. The tactics in studying the dependence on each factor is to vary this particular factor while all other variables are kept fixed.

\subsection{Varying the Area Ratio of False Lumen to True Lumen}

The first scenario is to vary the area ratio by altering the length scale $\mathrm{T}$ and $\mathrm{F}$ as defined in Figure 2(a). As illustrative example for discussion, values of $\mathrm{T}=12.5 \mathrm{~mm}$ and $\mathrm{F}=15.5 \mathrm{~mm}$ will result in an area ratio of false lumen to true lumen of 2:1. By steadily increasing $F$ (or decreasing T, Figure 2(a)), Figure 4(a) shows that the region of complete thrombosis shrinks, i.e. values of the $y$-coordinate of the flow cutoff plane move towards the origin. In other words, a smaller true lumen will imply a smaller domain of thrombosis. A typical flow configuration of this 'backflow' in the false lumen, after the entry

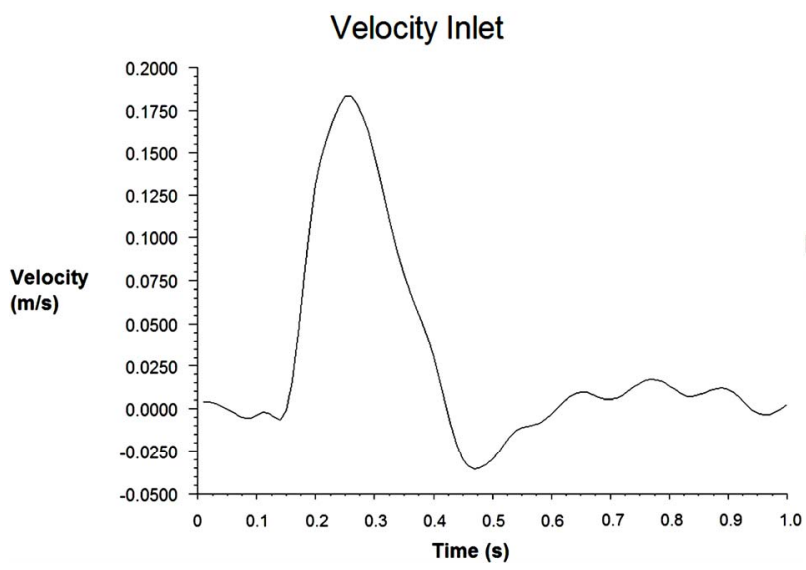

tear has been concealed by endovascular repair, shows indeed a large domain of stagnant fluid (Figure 4(b)).

The clinical implication is that patients with a larger false lumen are at a higher risk, as the domain of complete blood clot is now lesser in extent.

\subsection{Varying the Size of the Re-Entry Tear}

Another clinical consideration is the size of the re-entry tear, where typical range of $100 \mathrm{~mm}^{2}$ to $300 \mathrm{~mm}^{2}$ is chosen (Figure 2(b)). Figure 5 clearly shows a decreasing domain of complete thrombosis with increasing size of the distal re-entry tear, i.e. $y$ values of the cutoff plane move towards the origin.

These results conform to our intuition and the fluid physics. The smaller the re-entry tear, the smaller the flow rate into the false lumen will be, due to the decreasing area as well as the increased viscous resistance with the shrinking linear dimension.

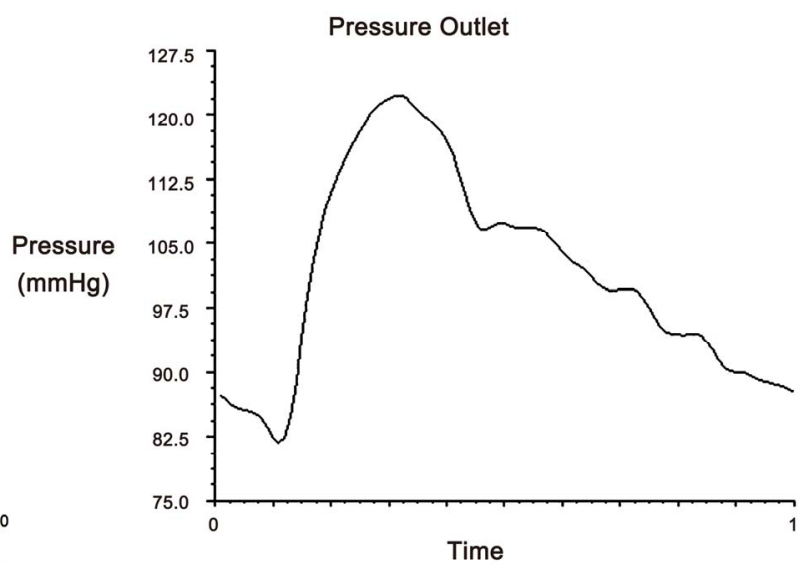

Figure 3. The waveforms for the pulsatile velocity inlet and the pulsatile pressure outlet.

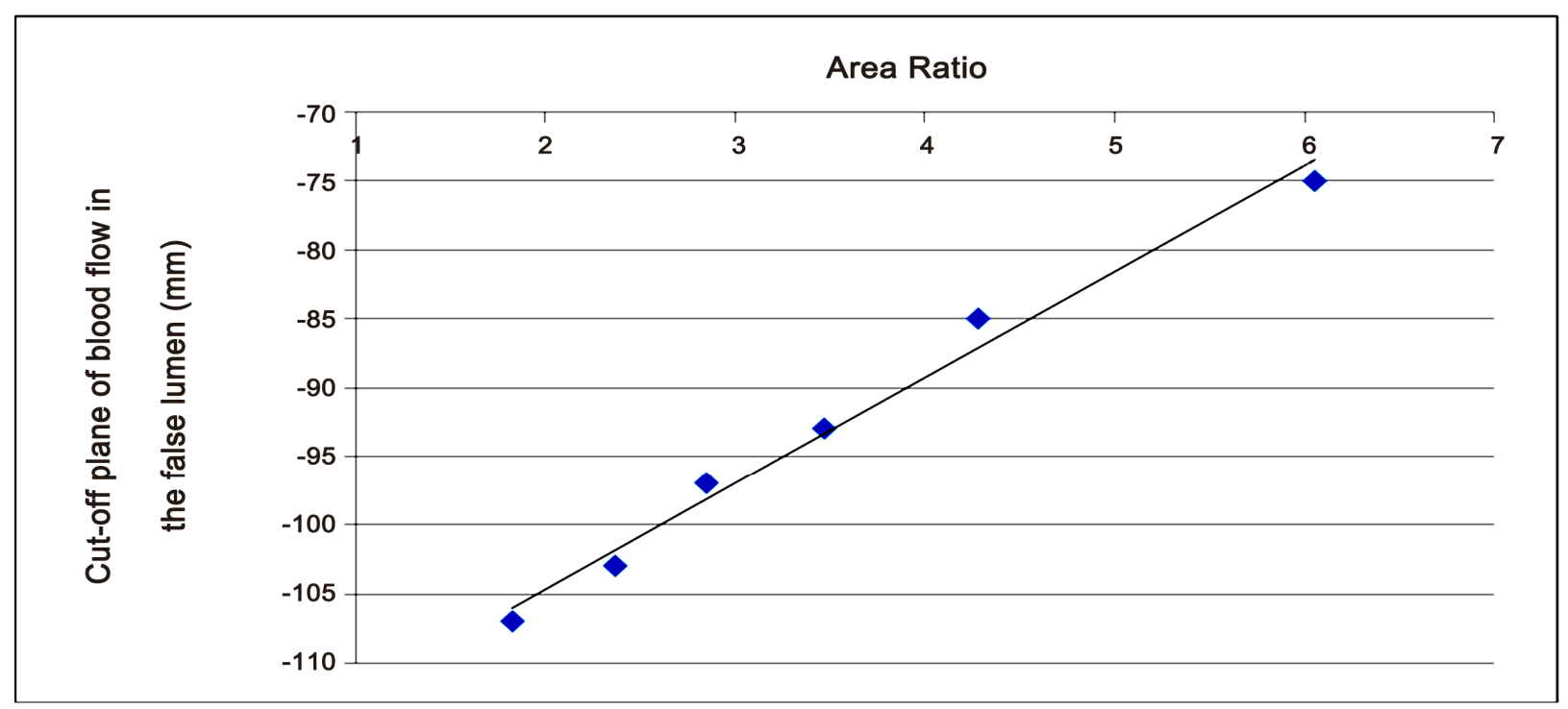

(a) 


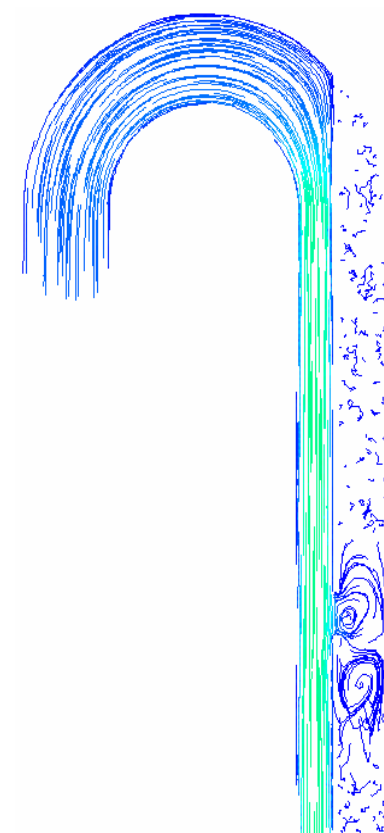

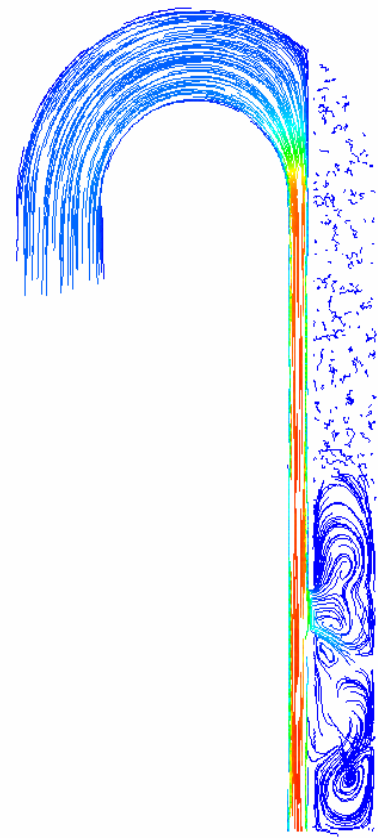

(b)
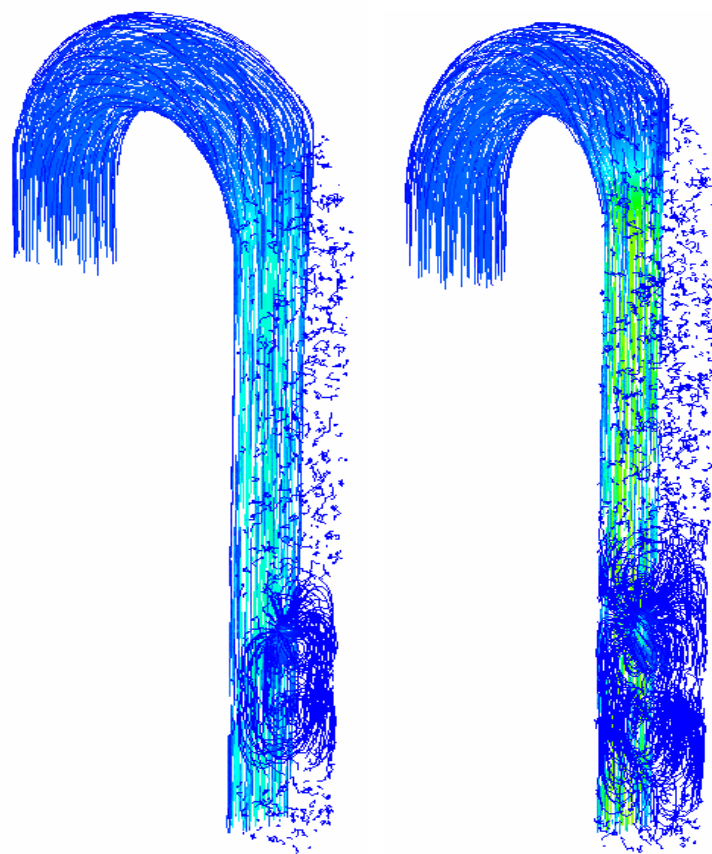

(c)

Figure 4. (a) Varying the area ratio of false lumen to true lumen; (b) After endovascular repair, a larger true lumen will have a larger extent of thrombosis (left), while a larger false lumen will have more back flow and smaller region of thrombosis (right). (Color code: Red = higher velocity; Blue = lower velocity); (c) three dimensional streamline plots in the dissected thoracic aorta after endovascular repair. A larger true lumen will have a larger extent of thrombosis (left), while a larger false lumen will have more back flow and smaller region of thrombosis (right). (Color code: Red = higher velocity; Blue = lower velocity).

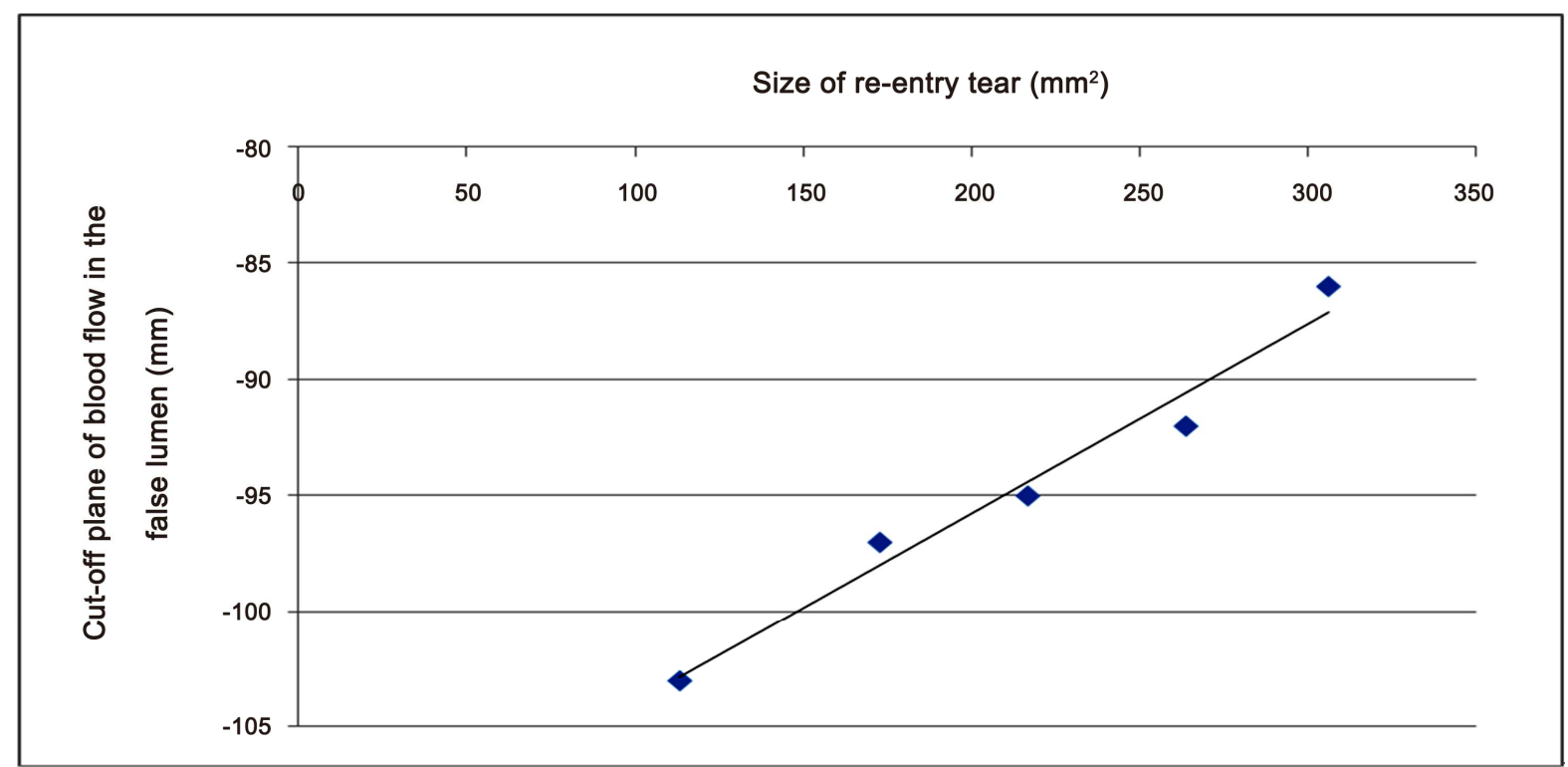

Figure 5. The relation between the size of the re-entry tear of the false lumen and the extent of complete thrombosis.

\subsection{Position of the Re-Entry Tear}

An examination of a large group of patients with TAD reveals that the position of the re-entry tear along the de- scending thoracic aorta will vary. Hence it is instructive to perform a numerical simulation in this direction. Figures 6(a), 6(b) show that the dependence on the position 


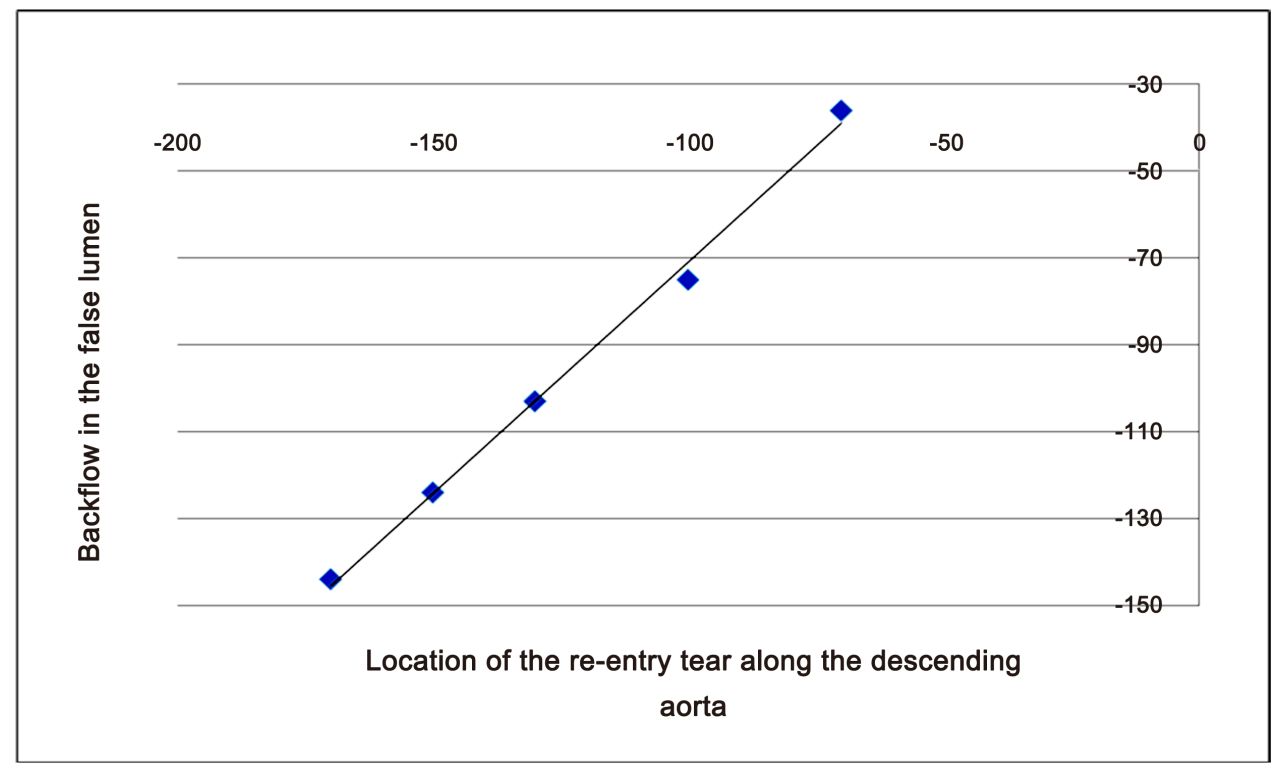

(a)

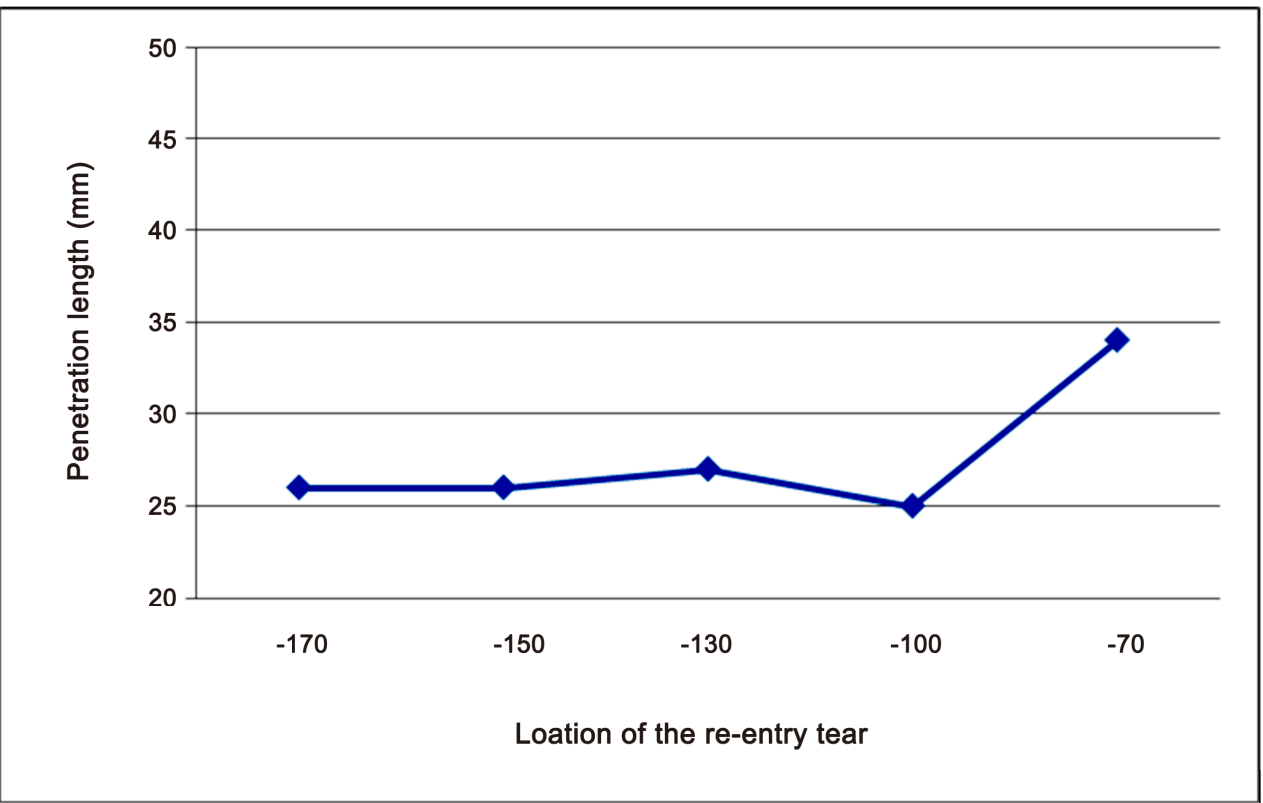

(b)

Figure 6. (a) Flow in the false lumen after endovascular repair: Effects of the location of re-entry tear on the backflow in the false lumen, area ratio (false to true lumens) $=2.37$, size of re-entry tear $=$ $88 \mathrm{~mm}^{2}$ (elliptical region of size $14 \mathrm{~mm}$ by $8 \mathrm{~mm}$ ); (b) Penetration length (region with blood flow) versus the position of the re-entry tear.

is mild, as the 'penetration length' is almost independent of the position of the re-entry tear. Here the penetration length is defined to be the depth of fluid in the false lumen where appreciable fluid motion exists, or more explicitly, as the distance between the cutoff plane and the reentry tear. The region of stagnant blood above this penetration length is assumed to achieve complete thrombo- sis.

\section{CONCLUSIONS}

Techniques and software from computational fluid dynamics (CFD) are used to study the problem of dissection along the thoracic aorta. The clinical practice of endovascular stent graft placement is still developing. Large scale studies and data collection are still ongoing efforts 
[17-19]. CFD study is appealing, as the cost is relatively low, and obviously poses no risk to the patients.

The main goals of this work are to help the clinicians (a) to assess the potential of rupture of the false lumen, and (b) to determine the need of undertaking secondary procedure. CFD [20-23] is employed here to assess the extent of thrombosis in the false lumen after endovascular repair, and the working assumption is that an absence of flow will lead to complete thrombosis of the blood.

After consultation with clinicians, three main biomechanical factors are identified and investigated. Firstly, the dependence on the area ratios of the lumens is studied. The main result is that patients with a smaller false lumen should be at a lower risk, as the domain of stagnant fluid / complete thrombosis is larger.

Secondly, a larger re-entry tear typically leads to larger region of blood in motion. This is consistent with fluid physics, as a smaller aperture means larger viscous resistance in the flow through re-entry tear, and thus a smaller flow rate. Consequently, a larger re-entry tear is probably undesirable. Thirdly, the extent of blood in motion is almost independent of the position of the re-entry tear along the descending aorta.

In conclusions, the area ratio of the lumens and the size of the re-entry tear are thus critical factors. These are indeed features vascular surgeons study from the computed tomography (CT) images of the patients. Hence CFD studies should complement clinicians' assessment of the risk and treatment procedures of the patients.

Finally, further research on

a) other biomechanical factors, e.g. varying blood pressure and modeling of a partially patent false lumen, and

b) improving the fluid physics modeling, e.g. incorporating non-Newtonian effects will definitely generate new scientific results and improve the management of this cardiovascular disease $[24,25]$.

\section{REFERENCES}

[1] Pedley, T.J. (1980) The fluid mechanics of large blood vessels. Cambridge University Press, Cambridge.

[2] Fung, Y.C. (1997) Biomechanics: Circulation. 2nd Edition, Springer, Berlin.

[3] Morris, L., Delassus, P., Callanan, A., Walsh, M., Wallis, F., Grace, P. and McGloughlin, T. (2005) 3-D numerical simulation of blood flow through models of the human aorta. Journal of Biomechanical Engineering - Transactions of ASME, 127(5), 767-775.

[4] Ricotta. J.J., Pagan, J., Xenos, M., Alemu, Y., Einav, S. and Bluestein, D. (2008) Cardiovascular disease management: The need for better diagnostics. Medical \& Biological Engineering \& Computing, 46(11), 1059-1068.

[5] El Zahab, Z., Divo, E. and Kassab, A. (2010) Minimization of the wall shear stress gradients in bypass grafts anastomoses using meshless CFD and genetic algorithms optimization. Computer Methods in Biomechanics and
Biomedical Engineering, 13(1), 35-47.

[6] Hassani, K., Navidbakhsh, M. and Rostami, M. (2007) Modeling of the aorta artery aneurysms and renal artery stenosis using cardiovascular electronic systems. Biomedical Engineering Online, 6(1), 22-31.

[7] Qiao, A. and Liu, Y. (2008) Medical application oriented blood flow simulation. Clinical Biomechanics, 23(Suppl 1), 130- 136.

[8] Hong, J., Wei, L., Fu, C. and Tan, W. (2008) Blood flow and macromolecular transport in complex blood vessels. Clinical Biomechanics, 23(Suppl 1), 125-129.

[9] Molla, M.M., Paul, M.C. and Roditi, G. (2010) LES of additive and non-additive pulsatile flows in a model arterial stenosis. Computer Methods Biomechanics and Biomedical Engineering, 13(1), 105-120.

[10] Cigarroa, J.E., Isselbacher, E.M., DeSanctis, R.W. and Eagle, K.A. (1993) Diagnostic-Imaging in the evaluation of suspected aortic dissection-Old standards and new directions. New England Journal of Medicine, 328(1), 35-43.

[11] Nienaber, C.A., Fattori, R., Lund, G., Dieckmann, C., Wolf, W., von Kodolitsch, Y., Nicolas, V. and Pierangeli, A. (1999) Nonsurgical reconstruction of thoracic aortic dissection by stent-graft placement. New England Journal of Medicine, 340(20), 1539-1545.

[12] Dake, M.D., Kato, N., Mitchell, R.S., Semba, C.P., Razavi, M.K., Shimono, T., Hirano, T., Takeda, K., Yada, I. and Miller, D.C. (1999) Endovascular stent-graft placement for the treatment of acute aortic dissection. New England Journal of Medicine, 340(20), 1546-1552.

[13] Tsai, T.T., Schlicht, M.S., Khanafer, K., Bull, J.L., Valassis, D.T., Williams, D.M., Bergner, R. and Eagle, K.A. (2008) Tear size and location impacts false lumen pressure in an ex vivo model of chronic type B aortic dissection. Journal of Vascular Surgery, 47(4), 844-851.

[14] Tsai, T.T., Evangelista, A., Nienaber, C.A., Myrmel, T., Meinhardt, G., Cooper, J.V., Smith, D.E., Suzuki, T., Fattori, R., Llovet, A., Froehlich, J., Hutchison, S., Distante, A., Sundt, T., Beckman, J., Januzzi, J.L., Isselbacher, E.M. and Eagle, K.A. (2007) Partial thrombosis of the false lumen in patients with acute type B aortic dissection. New England Journal of Medicine, 357(4), 349-359.

[15] Giannakoulas, G., Giannoglou, G., Soulis, J., Farmakis, T., Papadopoulou, S., Parcharidis, G. and Louridas, G. (2005) A computational model to predict aortic wall stresses in patients with systolic arterial hypertension. Medical Hypothesis, 65(6), 1191-1195.

[16] Gao, F., Watanabe, M. and Matsuzawa, T. (2006) Stress analysis in a layered aortic arch model under pulsatile flow. Biomedical Engineering Online, 5, 25-35.

[17] Böckler, D., Schumacher, H., Ganten, M., von Tengg-Kobligk, H., Schwarzbach, M., Fink, C., Kauczor, H.U., Bardenheuer, H. and Allenberg, J.R. (2006) Complicati- ons after endovascular repair of acute symptomatic and chronic expanding Stanford type B aortic dissections. Jo- urnal of Thoracic and Cardiovascular Surgery, 132(2), 361- 368.

[18] Eggebrecht, H., Nienaber, C.A., Neuhäuser, M., Baumgart, D., Kische, S., Schmermund, A., Herold, U., Rehders, T.C., Jakob, H.G. and Erbel, R. (2006) Endovascular stent - graft placement in aortic dissection: A metaanalysis. European Heart Journal, 27(4), 489-498. 
[19] Nienaber, C.A., Kische, S., Zeller, T., Rehders, T.C., Schneider, H., Lorenzen, B., Bünger, C. M. and Ince, H. (2006) Provisional extension to induce complete attachment after stent-graft placement in type B aortic dissection: The PETTICOAT concept. Journal of Endovascular Therapy, 13(6), 738-746.

[20] Wen, C.Y., Yang, A.S., Tseng, L.Y. and Chai, J.W. (2010) Investigation of pulsatile flowfield in healthy thoracic aorta models. Annals of Biomedical Engineering, 38(2), 391-402.

[21] Marzo, A., Singh, P., Reymond, P., Stergiopulos, N., Patel, U. and Hose, R. (2009) Influence of inlet boundary conditions on the local haemodynamics of intracranial aneurysms. Computer Methods in Biomechanics and Biomedical Engineering, 12(4), 431-444.

[22] Balossino, R., Pennati, G., Migliavacca, F., Formaggia, L., Venezziani, A., Tuveri, M. and Dubini, G. (2009) Computational models to predict stenosis growth in carotid arteries: Which is the role of boundary conditions? Computer Methods in Biomechanics and Biomedical Engineering, 12(1), 113-123.

[23] Lam, S.K., Fung, G. S.K., Cheng, S. W.K. and Chow, K.W. (2008) A computational study on the biomechanic- al factors related to stent-graft models in the thoracic aorta. Medical \& Biological Engineering \& Computing, 46(11), 1129-1138.

[24] Xu, X.Y. and Collins, M.W. (1995) Numerical modeling of blood flow in compliant arteries and arterial bifurcations. In: Power, H. Ed., Biofluid Mechanics, Computational Mechanics Publications, Southampton, 55-93.

[25] Shibeshi, S.S. and Collins, W.E. (2005) The rheology of blood flow in a branched arterial system. Applied Rheology, 15(6), 398-405.

[26] Wang, D. and Bernsdorf, J. (2009) Lattice Boltzmann simulation of steady non-Newtonian blood flow in a 3D generic stenosis case. Computer and Mathematics in Applications, 58(5), 1030-1034.

[27] Khoynezhad, A., Donayre, C.E., Omari, B.O., Kopchok, G.E., Walot, I. and White, R.A. (2009) Midterm results of endovascular treatment of complicated acute type B aortic dissection. Journal of Thoracic and Cardiovascular Surgery, 138(3), 625-631.

[28] Ting, A.C.W., Cheng, S.W.K. and Ho, P. (2003) Endoluminal stent grafts for aortic diseases: Experience at a major teaching hospital in Hong Kong. Australian and New Zealand Journal of Surgery, 73(3), 100-104. 